\title{
An Algorithm for Labeling Edges of Hierarchical Drawings *
}

\author{
Konstantinos G. Kakoulis and Ioannis G. Tollis \\ Department of Computer Science \\ The University of Texas at Dallas \\ Richardson, TX 75083-0688 \\ email: kostant@utdallas.edu, tollis@utdallas.edu
}

\begin{abstract}
Let $G(V, E)$ be a graph, and let $\Gamma$ be the drawing of $G$ on the plane. We consider the problem of assigning text labels to every edge of $G$ such that the quality of the label assignment is optimal. This problem has been first encountered in automated cartography. Even though much effort has been devoted over the last 15 years in the area of automated drawing of maps, the Edge Label Placement (ELP) problem remains essentially unsolved. In this paper we investigate the ELP problem. We present an algorithm for the ELP problem more suitable for hierarchical drawings of graphs, but it can be adopted to many different drawing styles and still remain effective. Also, we present experimental results of our algorithm that indicate its effectiveness.
\end{abstract}

\section{Introduction}

The area of graph drawing has grown significantly in the recent years motivated mostly by applications in information visualization $[4,17]$. When visualizing information, it is essential to display not only the structure of the objects and their relationships, but also important information about them. In other words, it is important to associate text labels with graphical features. This problem is called automatic label placement and has applications in many areas including graph drawing and cartography. Also, the automatic label placement problem has been recognized as an important area of research by the ACM Computational Geometry Task Force [7].

Most of the research addressing the labeling problem has been focused on labeling features of geographical and technical maps. Christensen, Marks and Shieber present a comprehensive survey of algorithms for the labeling problem [3]. Significant progress has been made in solving the Node (point) Label Placement (NLP) problem which consists of labeling points in the plane so that no labels overlap points or other labels (see $[1,3,6,8,10,18,20]$ ). However, the Edge Label Placement (ELP) problem remains essentially unsolved [14, 20]. It is worth noting that both the NLP $[8,13,15]$ and ELP [12] problems are NP-Hard.

\footnotetext{
* Research supported in part by NIST, Advanced Technology Program grant number 70 NANB5H1162. A patent on these and related results is pending.
} 
It has been suggested $[3,14]$ that the techniques used to solve the NLP problem can be used to solve the ELP problem by positioning a number of points on each edge, and then solving the NLP problem on those points. However, choosing the correct points is almost as hard as solving the original problem. Hence, no efficient techniques for the ELP problem are known to take advantage of the algorithms that solve the NLP problem.

Because the ELP problem is NP-Hard [12], any effective approach to solve the ELP problem must be directed towards devising heuristics. In this paper we first define the ELP problem in a formal way in Section 2. In Section 3 we present the main ideas of our algorithm. In Section 4 we present some improvements that can make the main algorithm more efficient. In Section 5 we present experimental results of an implementation of our algorithm, and we conclude in Section 6 .

\section{The ELP problem}

A drawing of a graph $G(V, E)$ is a representation of $G$ in the plane, where nodes are represented by symbols such as circles or boxes, and each edge $(u, v)$ is represented by a simple open curve between the symbols associated with the nodes $u$ and $v$. A straight - line drawing maps each edge into a straight-line segment. A polyline drawing maps each edge into a polygonal chain. A hierarchical drawing is a polyline drawing where the nodes and bends are constrained to lie on a set of equally spaced horizontal (vertical) lines, called layers. Next, we introduce some terminology specific to the ELP problem: (i) $\Lambda_{\varepsilon}$ is the set of all label positions for edge $e$; (ii) $\Lambda$ is the set of all label positions for all edges; (iii) $\lambda: E \rightarrow A$ is a function that assigns to edge $e$ in $E$ one label position from $A$, and $\lambda(e)=\lambda_{e} \in \Lambda_{e}$. Next, we define the ELP problem for a general drawing of a graph.

ELP is the problem of assigning text labels to each edge of a given drawing of a graph. The goal is to communicate the information for each edge via text labels in the best possible way, by positioning the labels in the most appropriate place. Here we consider the problem of assigning one label to each edge of the graph.

Cartographers Imhof [11] and Yoeli [19], have devised general rules that measure the quality of a label assignment.

Basic rules for labeling quality $[11,19]$ :

1. No overlaps of a label with other labels or other graphical features of the layout are allowed.

2. Each label can be easily identified with exactly one graphical feature of the layout (i.e., the assignment is unambiguous).

3. Each label must be placed in the best possible position (among all acceptable positions).

The first 2 rules evaluate how clear the association of the labels must be when compared with their corresponding edges. A label respects the first rule if it does not overlap any graphical feature, even though it is allowed to touch the edge 
that it belongs to. Also, a label respects the second rule if it is placed very close or touches the edge that it belongs to. The third rule defines a ranking among all label positions that typically captures the aesthetic preference for specific labels. This is an essential criterion for the quality of labeling of geographical and to some extend technical maps. It also allows to introduce problem specific constraints (i.e., the label of an edge must be closer to the source or destination node).

Each label position that is part of a final label assignment is associated with a cost. COST $: \Lambda \rightarrow \mathcal{N}$ is a function that gives us the cost of assigning label $\lambda_{e}$ to edge $e$ in the final label assignment. The $\operatorname{COST}\left(\lambda_{e}\right)$ is a linear combination of: (i) The cost with respect to the ranking of label $\lambda_{e} ;$ (ii) The cost which reflects the severity of the violation of the first two basic rules for label $\lambda_{e}$.
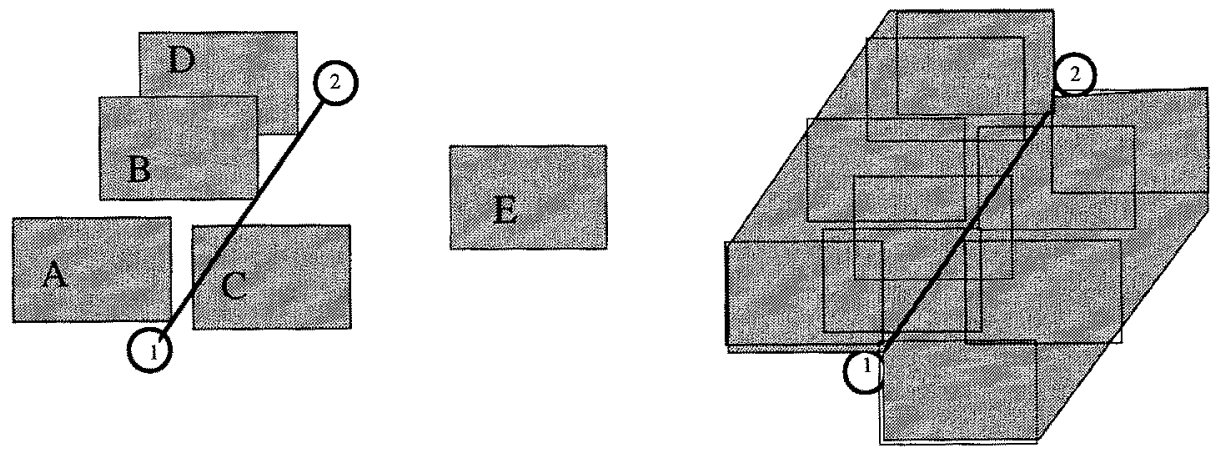

Fig. 1. (a) How to position labels for an edge. (b) Labeling space of an edge.

In Fig. 1 $(a)$ labels like $A, B$ or $D$ are preferable but certainly labels like $C$, which overlaps its associated edge, should be avoided. However, they can be acceptable with some appropriate cost assigned to them. If a drawing has more than one edges, then labels like $E$ (that float between edges) violate the second rule, and must be avoided.

We define the set of all label positions for a given edge either by explicitly defining a set of label positions (discrete labeling space) or by defining an area where labels can be placed (continuous labeling space). In the discrete labeling space, the set of all label positions is finite and each label is identified by its position in the drawing, as shown in Fig. 1(a). In the continuous labeling space, the set of all label positions is infinite, and for each edge we define a region which is bounded by a closed line, such that each label position for that edge must be placed inside that region, as shown in Fig. 1(b).

The ELP problem is an optimization problem since the objective is a label assignment of minimum cost. The objective is to find a set of labels, one for each edge, that yields minimum total cost. 
The ELP Problem : Let $G(V, E)$ be a graph and let $\Gamma$ be a drawing of $G$. Question: Find a label assignment that minimizes the following function:

$$
\sum_{i \in E} \sum_{j \in \Lambda_{2}} \operatorname{COST}(\lambda(i)) P(i, j)
$$

where:

$$
P(i, j)= \begin{cases}1, & \text { if } \lambda(i)=j, \\ 0, & \text { otherwise }\end{cases}
$$

and

$$
\sum_{i \in E} \sum_{j \in A_{i}} P(i, j)=|E|
$$

where:

$$
\sum_{j \in A_{i}} P(i, j)=1, \quad i \in E .
$$

\section{Solving the ELP problem}

In this section we present the main idea of our algorithm. Our technique works for labels that are parallel to the horizontal axis, and have approximately equal height. In order to simplify our discussion we make the following assumptions:

1. All labels have the same size.

2. Each edge has only one label associated with it.

However, our technique can be easily modified to work for labels of various sizes. Any acceptable solution for the ELP problem must guarantee at least two conditions:

1. Any label must be free of overlaps (except that it may overlap its associated edge).

2. Any label must be very close to its associated edge.

The goal of our algorithm is to assign to each edge a label that is free of overlaps and touches only its associated edge. The main idea of our algorithm is the following:

We divide the input drawing into consecutive horizontal strips of equal height. The height of each strip is equal to the height of the labels. Next we find the set of label positions $A_{e}$ for each edge $e$. Each label position must be inside a horizontal strip. We slide labels inside each horizontal strip, if a label touches an edge $e$, and it does not overlap any other edge and/or node, then we assign it as a label position of edge $e$, as shown in Fig. 2(a). In Fig. 2(b) the drawing is divided into horizontal strips, and the maximum number of label positions that follow the rules of our algorithm have been assigned to the edges of the drawing. As it can be observed from that figure, labels that overlap nodes or edges of the layout are not considered. Also labels are not allowed to intersect their associated edges. Labels must lie entirely into a horizontal strip. Thus, labels can only overlap other labels that belong to the same horizontal strip. Hence, the following lemma is true: 
Lemma 1. Each label position overlaps at most one other label position.
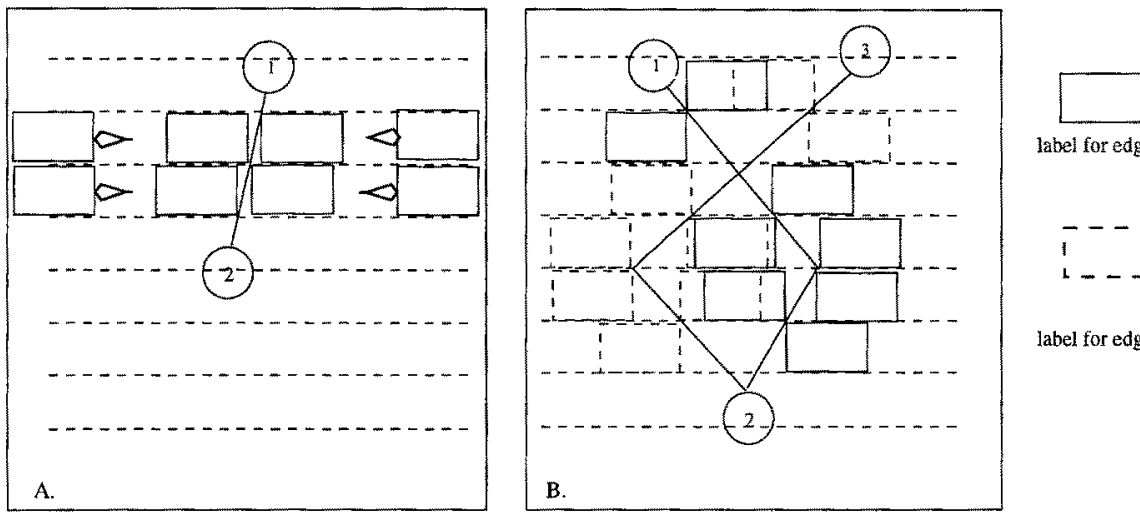

label for edge $(1,2)$
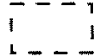

label for edge $(2,3)$

Fig. 2. Assigning label positions to edges of a drawing.

We transform the ELP problem into a matching problem where each edge $e$ is matched with one of its label positions in $\Lambda_{e}$. First, we group label positions together. If two label positions overlap then they belong to the same group. If a label position is free of overlaps then it belongs to a single member group. When we match edges to label positions, only one label position of each group can be part of a label assignment. This restriction guaranties a label assignment where labels do not overlap each other. Let $\mathcal{R}$ be the set of all such groups.

To further clarify the main idea of our algorithm we introduce the matching graph.

Definition 2. Given a drawing $\Gamma$ of a graph $G(V, E)$, and the set $A$ of label positions for $\Gamma$ (where each label position in $\Lambda$ overlaps at most one other label position), then a matching graph $G_{m}\left(V_{e}, V_{g}, E_{m}\right)$, is defined as follows:

- Each node $e$ in $V_{e}$ corresponds to an edge $e$ in $G$.

- Each node $r$ in $V_{g}$ corresponds to a group $r$ of label positions in $\mathcal{R}$.

- Each edge $(e, r)$ in $E_{m}$ connects a node $e$ in $V_{e}$, to a node $r$ in $V_{g}$, if and only if edge $e$ in $G$ has a label position that belongs to group $r$ of $\mathcal{R}$.

By definition graph $G_{m}$ is bipartite. In addition, the cost of assigning label $\lambda_{e}$ to edge $e$ may be the weight of edge $(e, r)$ in $G_{m}$, where $\lambda_{e} \in r$. Then, a maximum cardinality, minimum weight matching for graph $G_{m}$ will give us a label assignment of minimum cost (among all assignments) such that at most one label position from each group in $\mathcal{R}$ is part of that assignment. Hence, we have the following theorem: 
Theorem 3. Let $A$ be the set of label positions for all edges of a drawing $\Gamma$ of graph $G(V, E)$. If every label position in $A$ overlaps at most one other label position in $A$, then a maximum cardinality minimum weight matching of the corresponding matching graph $G_{m}$ produces an optimal solution to the ELP problem with no overlaps.

The result of Theorem 3 gives rise to a powerful technique for the solution of the ELP problem, given specific conditions on the set of label positions. In practice we can satisfy these conditions heuristically. The main problems that need to be solved heuristically are: $(i)$ Defining the best set $\Lambda$; $(i i)$ Partitioning $A$ into groups in $\mathcal{R}$. In our technique, we define the set $A$ of label positions by splitting the input drawing into horizontal strips, and assigning label positions to edges as we have explained at the beginning of this section. Also, according to Lemma 1, each label position overlaps at most one other label. Thus, it is trivial to construct the set of groups $\mathcal{R}$. Next, we give a description of the ELP algorithm.

\section{ELP_Algorithm}

INPUT: A drawing $\Gamma$ of graph $G(V, E)$.

OUTPUT: A label assignment.

1. Split $\Gamma$ into horizontal strips.

2. Find all label positions for each edge and construct the groups in $\mathcal{R}$.

3. Create the matching graph $G_{m}$ for $\Gamma$.

4. Match edges to label positions, by finding a matching in $G_{m}$.

The last step in the algorithm solves the maximum cardinality minimum weight matching problem for the matching graph. By Theorem 3 , the maximum cardinality minimum weight matching of the matching graph will give an optimal label assignment with respect to the set of labels $A$ that satisfy the sufficient conditions of Theorem 3 . We can use any known algorithm to find a maximum cardinality minimum weight matching of the matching graph $G_{m}$. Notice that the best algorithms take more than quadratic time with respect to the size of $G_{m}[9,16]$.

The size of the matching graph depends on the size of set $A$ of label positions. Unfortunately the size of $A$ can be very large with respect to the size of the original graph $G$. This implies that a typical matching algorithm might take a long time. Additionally, in our application graph $G_{m}$ has a rather simple structure. In order to reduce the time complexity of the matching we present in the next section a heuristic that finds a maximum cardinality matching with low total weight in linear time with respect to the size of $G_{m}$. Some important additional advantages of this heuristic are: reduced memory requirements, simplicity, and that it can be easily embedded into different systems.

\subsection{A fast matching heuristic.}

Here we present a fast heuristic that solves the maximum cardinality matching problem for the matching graph. The size of the matching graph depends on 
the size and the total edge length of the drawing, and not only on the size of the input graph. From the definition of the matching graph, and Lemma 1, it is clear that the matching graph is a bipartite graph such that each node in $V_{g}$ has degree at most 2.

The following algorithm finds a maximum cardinality matching for $G_{m}$.

\section{Fast_Matching Heuristic}

INPUT: Matching graph $G_{m}$.

OUTPUT: A maximum cardinality matching for $G_{m}$ with low total weight.

1. If the minimum weight incident edge of a node in $V_{e}$

connects this node to a node in $V_{g}$ of degree 1 then:

Assign this edge as a matched edge.

Update $G_{m}$.

2. If a node in $V_{e}$ has degree one then:

Assign its incident edge as a matched edge.

Update graph $G_{m}$.

3. Repeat Steps 1 and 2 until no new edge can be matched.

4. Delete all nodes of degree 0 from $G_{m}$.

For each node $e$ in $V_{e}$ do:

Remove all but the two incident edges of $e$ with the least weight.

5. The remaining graph consists of simple cycles and/or paths.

Find the only two maximum cardinality matchings for each component.

Choose the matching of minimum weight.

Note: The Update $G_{m}$ operation removes the two nodes incident to a new matched edge and stores that edge and its incident nodes as part of the matching. Also removes all incident edges from the two nodes.

In Step 1 we find matched edges that are part of any optimal solution. In Step 2 we find matched edges to those nodes in $V_{e}$ that are of degree one. If two nodes of degree one in $V_{e}$ are connected to the same node in $V_{g}$, we choose as matched edge the edge with minimum weight. This implies that one of the edges will have no label. Next, in Step 4, for each node in $V_{e}$ of degree more than two, we keep only its two incident edges of least weight and remove the rest of the edges. Now each node in $V_{e}$ has degree two and each node in $V_{g}$ has degree at most two. The remaining bipartite graph has a simple structure: It consists of simple cycles and/or simple paths. Each path or cycle has exactly two maximum cardinality matchings. It is trivial to find both of them by simply traversing the cycle or path and picking as part of the matching only the even or odd numbered edges.

Next, we show that this fast matching heuristic produces a maximum cardinality matching.

Theorem 4. The matching produced by the Fast Matching Heuristic is a maximum cardinality matching.

Sketch of proof: Let us assume that the matching produced by the matching heuristic is not a maximum cardinality matching. Then, there exists an aug- 
menting path according to Berge's theorem [2], which states that: A matching $\mathrm{M}$ of a graph $\mathrm{G}$ is a maximum matching if and only if $\mathrm{G}$ contains no augmenting paths.

Any augmenting path is of odd length, which implies that for graph $G_{m}$ any such path has one end in $V_{e}$ and the other end in $V_{g}$. So, without loss of generality, we assume that any augmenting path starts from a node $e$ in $V_{e}$ and ends in a node $g$ in $V_{g}$.

It is clear that $e$ has been deleted before Step 5 of the algorithm. Also, $g$ is connected only to nodes in $V_{e}$ that are matched in the last step of the algorithm. Thus, there exists at least one edge in the augmenting path that connects a node $u$ deleted before Step 3 to a node $v$ not deleted before Step 5 . In addition, that edge can only be a non-matched edge, otherwise both nodes would have been deleted (not deleted) before Step 5. A node $w$ in $V_{e}$ is deleted before Step 5 if it has degree 1 or 0 during the execution of the first three steps. Thus, node $w$ is always connected only to nodes deleted at the same step as $w$. Consequently, $u$ must be a node in $V_{g}$. We have arrived in a contradiction, because edge $(u, v)$ is an even numbered edge in the augmented path which implies that $(u, v)$ must be a matched edge. Thus, we have proved our claim.

The fast matching heuristic finds a maximum cardinality matching with low total weight because in the last step it considers only the two incident edges of nodes in $V_{e}$ with the lowest weight. In addition, if each edge in the input drawing has at least two label positions, the fast matching heuristic produces a label assignment that assigns to at least half of the edges their best label position, and to the rest of the edges their second best label position.

\section{Improvements and Extensions.}

One of the weaknesses of the algorithm presented in this paper is that it considers only a subset of all label positions. Specifically, label positions are defined by the horizontal strips that divide the drawing. Hierarchical drawings are particularly suitable for this algorithm since edges are usually long and almost vertical. If a hierarchical drawing is drawn from left to right then we rotate the drawing by 90 degrees and apply the algorithm.

In order to improve the effectiveness of our algorithm a postprocessing step is often necessary. The postprocessing step explores the solution space in three ways:

1. It locally shifts assigned labels to create space for a new label to be assigned.

2. It searches the solution space to find if there is enough space to assign a label with or without repositioning already assigned labels.

3. It searches the solution space after relaxing the restrictions on the quality of the label assignments by allowing labels to overlap their associated edges.

In the presentation of the algorithm we assumed that labels of different edges are of the same size. In reality, we only need to have labels that are of approximately equal height. Because in order to find the set of label positions for the 
input drawing we must divide the drawing into equally spaced horizontal strips. If labels have different heights, then if we assume that each label has height equal to the maximum height among all labels we can apply the present algorithm to solve the ELP problem.

One important characteristic of the ELP algorithm is that it can be tailored to take into account the user's preferred positions when it assigns labels to edges. The user can specify preferred positions to place labels (i.e., close to the source or target node), and by ranking the label positions according to her/his specifications, the algorithm will produce a label assignment that respects the user's preferred positions.

After extensive experimentation, we noticed that this algorithm performs very well not only for hierarchical drawings, but also for other straight-line drawings, such as drawings produced by force-directed and circular techniques. One weakness of the algorithm is that it ignores horizontal edges or edge segments. Thus, it is not suitable for orthogonal drawings. One can divide an orthogonal drawing into horizontal and vertical strips in order to find a suitable set of label positions and then use the rest of the algorithm to assign labels to edges.
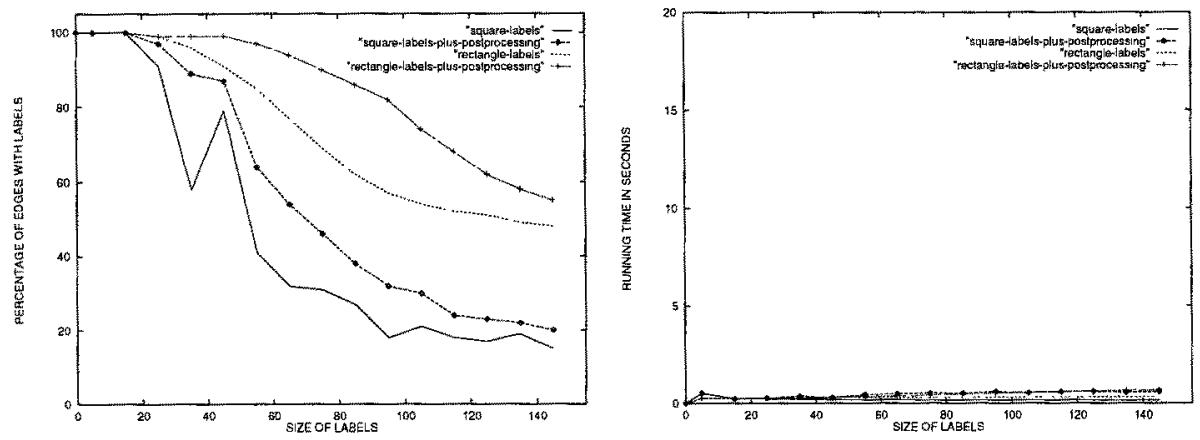

Fig. 3. Experimental results for graphs of 30 nodes and on the average 36 edges.

\section{$5 \quad$ Experimental results.}

We have implemented the ELP algorithm on top of the Graph Layout Toolkit $\left(\mathrm{GLT}^{2}\right)$ in $\mathrm{C}++$. We ran the experiments on a SPARC 5 unix based machine. We used a subset of graphs used in the experimental analysis of [5]. Actually, we used all graphs of $30,60,80,90$, and 99 nodes in [5].

The goal of our experiments was to give us an indication of the practical effectiveness of the algorithm presented here. It was not our goal to produce

${ }^{2}$ GLT is a product of Tom Sawyer Software (http://www.tomsawyer.com). 

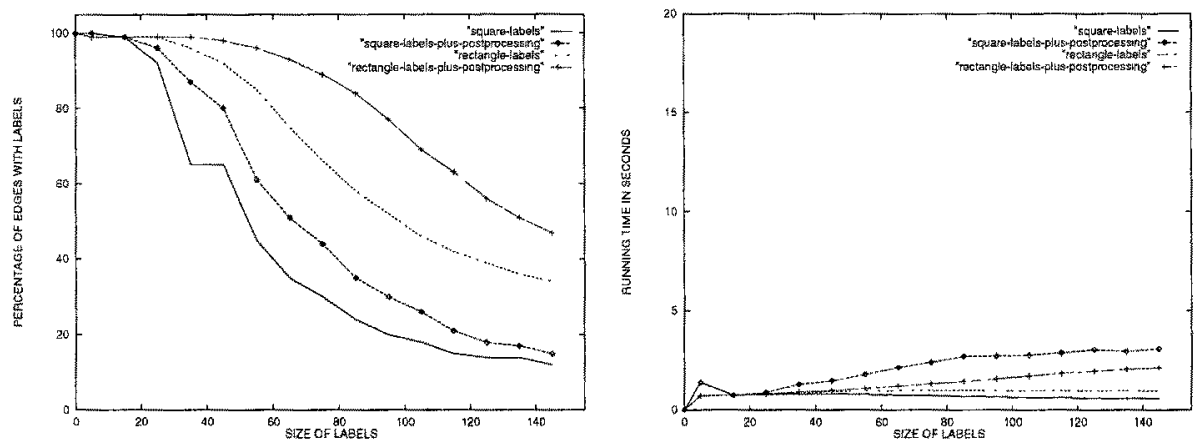

Fig. 4. Experimental results for graphs of 60 nodes and on the average 79 edges.

a comprehensive evaluation of the ELP algorithm, because it is very hard to quantify the complexity of a given drawing with respect to a label assignment. In our case, we drew the graphs using the hierarchical style algorithm implemented in GLT. We drew all graphs using the same tailoring options (i.e., minimum allowed separation of nodes in the same layer, size of nodes and distance between consecutive layers). We were more interested in how the success rate (percentage of edges with assigned labels) was decreasing as we were increasing the size of the labels.

After drawing the graphs, we ran the ELP algorithm with different size labels on the same drawings. We ran 2 sets of experiments; (i) Labels are square, and in successive runs of the algorithm we increase the size of the square; ( $i$ ) Labels are rectangles, and in successive runs of the algorithm we increase only the width of the label. In the final label assignment produced by the ELP algorithm each assigned label does not overlap any other label, edge, or node (even though it is allowed to overlap its associated edge). If we allow labels in the final assignment to overlap other graphical features in the drawing, then the success rate of the ELP algorithm is much greater. However, there will be a significant decrease in the quality of the label assignment.

In Figures 3 and 4 we present some results of our experiments. The plain lines represent the results after running only the main algorithm. The marked lines represent the results after running the algorithm with the postprocessing step. In each figure the left plot shows the success rate of the algorithm (the size of the labels is represented in the $\mathrm{x}$-direction, and the percentage of edges with assigned labels is represented in the $y$-direction). The right plot shows the running time of the same set of experiments that are represented in the left plot.

All drawings are grid drawings, that is nodes and bends have integer coordinates. The label size is with relation to the grid-size. For example, a label with width 20 is a label with width equal to 20 grid units.

A typical example of a hierarchical drawing with labels is shown in $F i g, 5(a)$, and a straight-line drawing (circular style) with labels is shown in Fig. $5(b)$. In those figures the dark rectangles are the labels. 

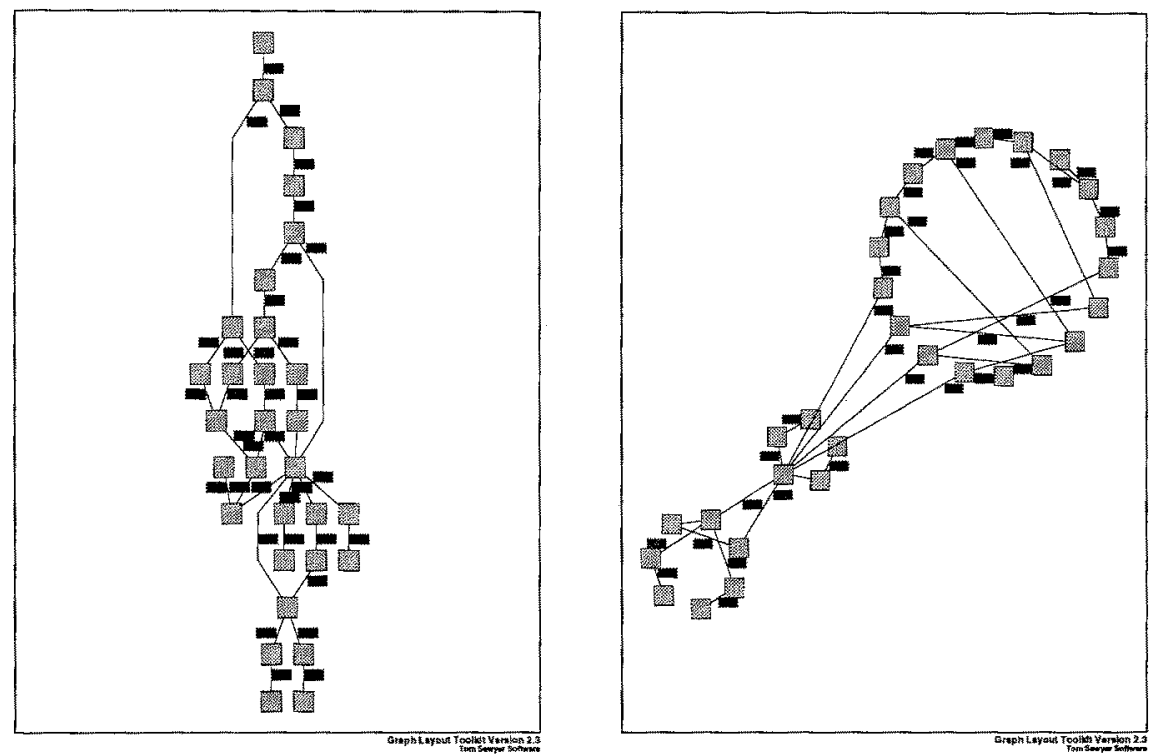

Fig. 5. Sample drawings with edge labels.

\section{Conclusions}

We have presented an algorithm for the ELP problem. Our experiments have shown that the approach to solve the ELP problem described in this paper is very successful, especially for hierarchical drawings. As we mentioned in the introduction, the ELP and NLP problems have been first proposed in the framework of automated cartography, where fixed geometry is one element that cannot be compromised. When labeling graphs, where the underlying geometry of the drawing is a result of a layout algorithm used to draw the graph, the labeling problem is and can be more flexible. It is an interesting problem to investigate how one can change the drawing of a graph to free up space that can be used to assign labels without compromising the quality of the drawing.

\section{Acknowledgements.}

We would like to thank Tom Sawyer Software, and especially its President and CEO Brendan Madden, for helpful discussions, and for making their software available to implement our labeling algorithms. 


\section{References}

1. J. Ahn and H. Freeman. A program for automatic name placement. Cartographica, 21(5 \& 3):101-109, Summer \& Autumn 1984.

2. C. Berge. Two theorems in graph theory. In Proc. Natl. Acad. Sci., 43, pages $842-844,1957$.

3. J. Christensen, J. Marks, and S. Shieber. An empirical study of algorithms for Point Feature Label Placement. ACM Trans. on Graphics, 14(3):203-232, July 1995.

4. G. Di Battista, P. Eades, R. Tamassia, and I. G. Tollis. Algorithms for drawing graphs: an annotated bibliography. Comput. Geom. Theory Appl., 4:235-282, 1994.

5. G. Di Battista, A. Garg, G. Liotta, R. Tamassia, E. Tassinari, and F. Vargiu. An experimental comparison of four graph drawing algorithms. Comput. Geom. Theory Appl., 1996. to appear.

6. L. R. Ebinger and A. M. Goulete. Noninteractive automated names placement for the 1990 decennial census. Cartography and Geographic Informaton Systems, 17(1):69-78, January 1990 .

7. ACM Computational Geometry Impact Task Force. Application challenges to computational geometry. Technical Report TR-521-96, Princeton Univ., 1996.

8. M. Formann and F. Wagner. A packing problem with applications to lettering of maps. In Proc. 7th Annu. ACM Sympos. Comput. Geom., pages 281-288, 1991.

9. A. V. Goldberg and R. Kennedy. An Efficient Cost Scaling Algorithm for the Assignment Problem. Mathematical Programming, 71:153-178, December 1995.

10. S. A. Hirsch. An algorithm for automatic name placement around point data. The American Cartographer, 9(1):5-17, 1982.

11. E. Imhof. Positioning names on maps. The American Cartographer, 2(2):128-144, 1975.

12. K. G. Kakoulis and I. G. Tollis. On the Edge Label Placement Problem. In S. North, editor, Graph Drawing (Proc. $G D$ '96), volume 1190 of Lecture Notes in Computer Science, pages 241-256. Springer-Verlag, 1997.

13. T. Kato and H. Imai. The NP-completeness of the character placement problem of 2 or 3 degrees of freedom. In Record of Joint Conference of Electrical and Electronic Engineers in Kyushu, pages 11-18, 1988. In Japanese.

14. J. Marks. Personal communication, 1996.

15. J. Marks and S. Shieber. The computational complexity of cartographic label placement. Technical Report 05-91, Harvard University, 1991.

16. R. E. Tarjan. Data Structures and Network Algorithms, volume 44 of CBMS-NSF Regional Conference Series in Applied Mathematics. Society for Industrial Applied Mathematics, 1983.

17. I. G. Tollis. Graph Drawing and Information Visualization. ACM Computing Surveys, 28A(4), 1996.

18. F. Wagner and A. Wolf, Map labeling heuristics: Provably good and practically useful. In Proc. 11th Annu. ACM Sympos. Comput. Geom., pages 109-118, 1995.

19. P. Yoeli. The logic of automated map lettering. The Cartographic Journal, 9(2):99-108, 121972.

20. S. Zoraster. The solution of large 0-1 integer programming problems encountered in automated cartography. Operation Research, 38(5):752-759, September-October 1990 . 